\title{
Point-of-Care Lung Ultrasound to Evaluate Lung Isolation During One-Lung Ventilation in Children: Narrative Review
}

This article was published in the following Dove Press journal:

Medical Devices: Evidence and Research

\author{
Yoshikazu Yamaguchi (iD ${ }^{1,2}$ \\ Alok Moharir ${ }^{1,2}$ \\ Candice Burrier ${ }^{1,2}$ \\ Takeshi Nomura ${ }^{3}$ \\ Joseph D Tobias $\mathbb{D}^{1,2}$ \\ 'Department of Anesthesiology \& Pain \\ Medicine, Nationwide Children's \\ Hospital, Columbus, OH, USA; \\ 2Department of Anesthesiology, The \\ Ohio State University, Columbus, $\mathrm{OH}$, \\ USA; ${ }^{3}$ Department of Intensive Care \\ Medicine, Tokyo Women's Medical \\ University, Tokyo, Japan
}

\begin{abstract}
This review focuses on the current technique and evidence regarding the use of point-of-care ultrasound (POCUS) to evaluate lung isolation for thorax surgery in infants and children. Previous reports in infants and children are presented. Figures and high-quality video are used to demonstrate the technique for POCUS in pediatric patients and to highlight differences between pediatric and patients. Lung sliding in B-mode and the seashore sign in M-mode suggest that the lung is ventilated. Pediatric anesthesiologists should be familiar with this technique as it is non-invasive and may also be more accurate when compared with auscultation.
\end{abstract}

Keywords: one-lung ventilation, point-of-care ultrasound, POCUS, thoracic surgery, lung ultrasound, ultrasonography

\section{Introduction}

Effective one-lung ventilation (OLV) is essential for successful video-assisted thoracic surgery in children as it allows exposure of the operative field. Failure of the technique has been reported as the most common cause for the need to convert to open thoracotomy. ${ }^{1}$ There are several ways to confirm lung separation, including ultrasound, auscultation, visual examination, and fiberoptic bronchoscopy. Although fiberoptic bronchoscopy is the gold standard, it may be difficult to ventilate the patient during the procedure especially in an infant or small child.

Lung ultrasound has been shown to have a higher diagnostic accuracy than auscultation in determining tracheal versus bronchial intubation in adults. ${ }^{2}$ Similar results have been reported when using a double lumen endotracheal tube for $\mathrm{OLV}^{3-6}$ To date, there are a limited number of case reports regarding the use of lung ultrasound in children and no prospective clinical trials. There is increasing clinical use of point-of-care ultrasound (POCUS) to identify effective lung separation thereby eliminating the need for bronchoscopy and limiting inaccuracies with auscultation. Confirmation of lung separation using point-of-care ultrasound can be performed with both the Brightness-mode (B-mode) and Motion-mode (M-mode). Lung sliding and lung pulse are used in B-mode while the seashore sign and the bar-code sign are important in M-mode. ${ }^{7,8}$

Therefore, there appears to be a need to increase the understanding of the applications of lung ultrasonography in clinical practice in infants and children.
Correspondence: Yoshikazu Yamaguchi Department of Anesthesiology \& Pain Medicine, Nationwide Children's Hospital, 700 Children's Drive, Columbus, $\mathrm{OH}$ 43205, USA

Tel + I $614722-4200$

Fax +I 614 722-4203

Email yoshikaz@rd6.so-net.ne.jp
Medical Devices: Evidence and Research 2020:13 385-389

DovePress in $\mathbf{D}$ 
A systematic search of PubMed ${ }^{\circledR}$ was conducted through September 2020 using a combination of controlled vocabulary (MeSH) and free text terms (Supplementary Table 1). The abstracts from the publications were reviewed and those pertaining to pediatric-aged patients were included for further review. Additionally, the reference list of these publications was reviewed to ensure that all pediatric reports regarding the use of POCUS involving thoracic surgery and OLV had been identified.

\section{Previous Publications}

We identified 72 articles which fit our search strategy. Upon further review, we identified 4 case reports and 2 letters to the editor which were associated with lung ultrasound and one-lung ventilation in infants and children. ${ }^{7-12}$ One case report and one letter were written by the authors of this manuscript. Our letter was in response to one of the case reports and pointed out a potential misunderstanding of the images reported. Point-of-care lung ultrasound to confirm one lung ventilation is a developing field. The current review describes out a technique for POCUS of the lung and its applications during OLV in infants and children.

\section{Equipment and Preparation}

The patient is positioned in the supine position. After placement of the device for OLV such as a double lumen endotracheal tube or bronchial blocker, lung separation can be confirmed by point-of-care ultrasound. A high-frequency linear probe $(5-13 \mathrm{MHz})$ with standard lung settings is best to evaluate the pleural membrane and its artifacts in children. The probe should be covered with a plastic cover with standard ultrasound gel inside and outside the cover. In general, medical ultrasound uses the convention that the orientation marker should correspond to the left side of the screen. The depth is adjusted so that the pleural membrane is $1 / 2$ nd to $1 / 3 \mathrm{rd}$ of the distance of the screen from the top.

\section{Procedure}

\section{Confirmation by B-Mode}

Scans are longitudinal. The probe is placed in the midclavicular line between the second and the third intercostal space or at the mid-axillary line between the 4th and 5th intercostal spaces. ${ }^{13,14}$ In B-mode, two types of movement can be seen at the pleural line: lung sliding and lung pulse. Both lung sliding and lung pulse are a horizontal movement at the pleural line. Lung sliding is a movement in rhythm with respiration, indicating sliding of the visceral pleura against the parietal pleura (Supplementary Video 1$).{ }^{13}$ Lung pulse is a smaller and faster movement than lung sliding (Supplementary Video 2), because lung pulse is generated by the heartbeat (Table 1). ${ }^{15}$ Lung pulse is usually easy to see and faster in small children than in adults.

Lung POCUS begins with identification of the Bat sign, the most basic image of lung ultrasound (Figure 1A and $\mathrm{B}$ ). One will be able to see the pleural line under the rib in infants and small children because of immature ossification of the bones. Once this view is obtained, focus on the pleural line without ventilating the patient to see lung pulse. Then, ventilate the patient and identify lung sliding, which is identified at the pleural line. The sound of the pulse oximeter is helpful to differentiate lung sliding from lung pulse. If the lung is collapsed such as during OLV, neither lung sliding, nor lung pulse will be seen. These are the same findings that are seen in the presence of a pneumothorax. What you can see are multiple A-lines which are reverberation artifacts of the pleural line.

\section{Confirmation by M-Mode}

The seashore sign and the bar-code sign (stratosphere sign) are important findings in M-mode (Figures 2-4). Again, one needs to identify the Bat sign as in the B-mode. The M-mode scanning line must be set on the pleural line, not on the rib. If the lung is ventilated, the seashore sign will be seen with the M-mode (Figure 2). If the lung is not ventilated, the bar-code sign will be seen. There may be small artifacts in bar-code sign at regular intervals, which are generated by the lung pulse (Table 2). M-mode is more helpful in older patients than in infants and young children because a higher heart rate creates a lot of noise in the Barcode sign which may be misinterpreted as Sea-shore sign (Figures 3 and 4).

Table I Lung Sliding and Lung Pulse

\begin{tabular}{|l|l|l|}
\hline & Lung Sliding & Lung Pulse \\
\hline Direction & Horizontal & Horizontal \\
Frequency & Respiratory rate & Heart rate \\
Movement & Large & Small \\
\hline
\end{tabular}




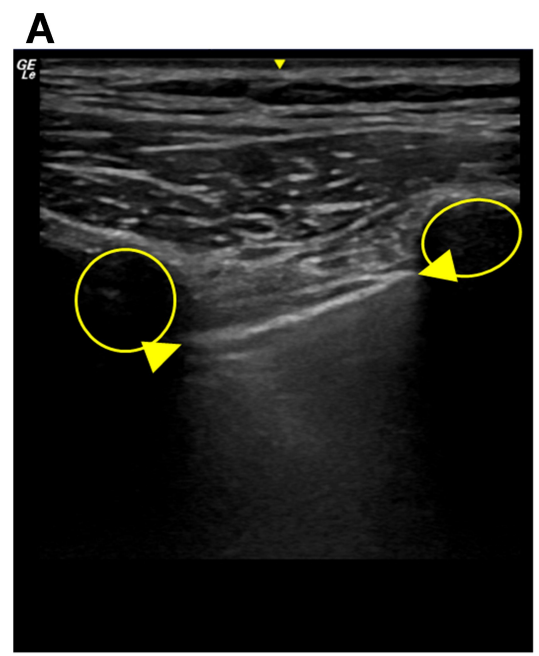

\section{B}

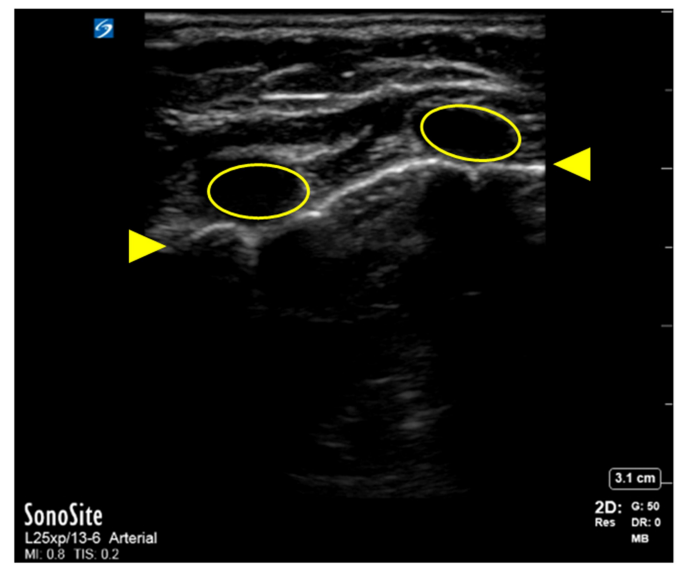

Figure I Demonstration of the Bat sign in an adult patient $(\mathbf{A})$ and an infant $(\mathbf{B})$. The Bat sign is the most basic view of lung ultrasound. The ribs are identified as low echoic regions (circles) with an acoustic shadow. The pleural line (between arrows) is a high echogenic (white) line just below and between the two ribs. Above the pleural line is subcutaneous tissue. All of the findings below the pleural line are artifact.

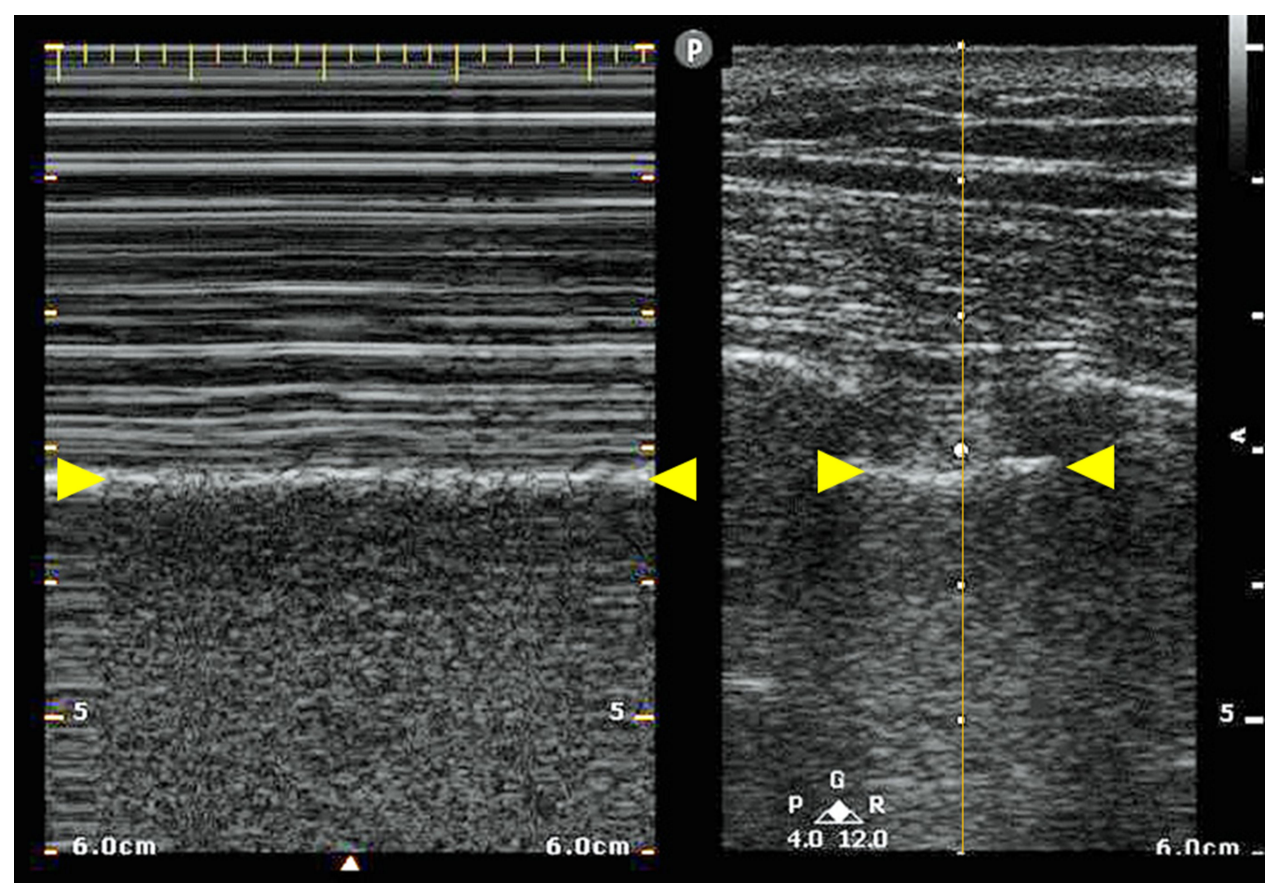

Figure $2 \mathrm{M}$-mode (left image) and B-mode (right image). On the right image, the M-mode shooting line is in the middle of the picture (circle and yellow line). Above the pleural line (yellow arrows), there are several horizontal lines. A sandy pattern below the pleural line is known as the seashore sign, which is generated by lung sliding. This confirms that the lung is ventilated.

\section{Keys to Success}

1. The Bat sign is the first view to obtain as it helps one find the pleural line.

2. Manual ventilation makes it easier to differentiate lung sliding from lung pulse.

3. Try to avoid including the heart in the image because cardiac motion will make it difficult to focus on the pleural line.
4. M-mode should be evaluated when the patient is ventilated.

5. Lung pulse with a high heart rate may lead to the barcode sign being misinterpreted as the sea-shore sign.

\section{Limitations of Lung Ultrasonography}

Although it has high diagnostic accuracy in several clinical scenarios, POCUS of the lung has specific limitations. There 
Table 2 Ultrasound Findings in B-Mode and M-Mode

\begin{tabular}{|l|l|l|l|}
\hline Mode & & Ventilated & Non-Ventilated \\
\hline B-mode & $\begin{array}{l}\text { Lung pulse } \\
\text { Lung sliding }\end{array}$ & $\begin{array}{l}\text { Present } \\
\text { Present }\end{array}$ & $\begin{array}{l}\text { Present or absent } \\
\text { Absent }\end{array}$ \\
\hline M-mode & & Seashore sign & Bar-code sign \\
\hline
\end{tabular}

is an initial investment required for the purchase of the machine. Lung ultrasonography is point-of-care and provides only an instantaneous view. As such, intermittent scanning may be required during surgery. Although lung ultrasonography is not difficult, training and continued practice is necessary. Finally, there are several situations which decrease the diagnostic accuracy of lung ultrasound. Lung ultrasound is based not only on the anatomical structure, but also on the interpretation of the artifact, which is made by a combination of the visceral pleura and the parietal pleura.

Various pathologic processes or conditions can interfere with the use of lung ultrasound. Pneumothorax is a well-known cause of absent lung sliding. ${ }^{16}$ In patients with pneumothorax, the parietal pleura is not attached to the visceral pleura. Therefore, the ultrasound beams are reflected at the parietal pleura with the production of multiple reverberation artifacts known as a-lines. ${ }^{17,18}$ As air in the thoracic cavity is generally trapped toward the apex, if both lung sliding and lung pulse are not visible, the dependent areas should be scanned to confirm whether the lung is ventilated or not.

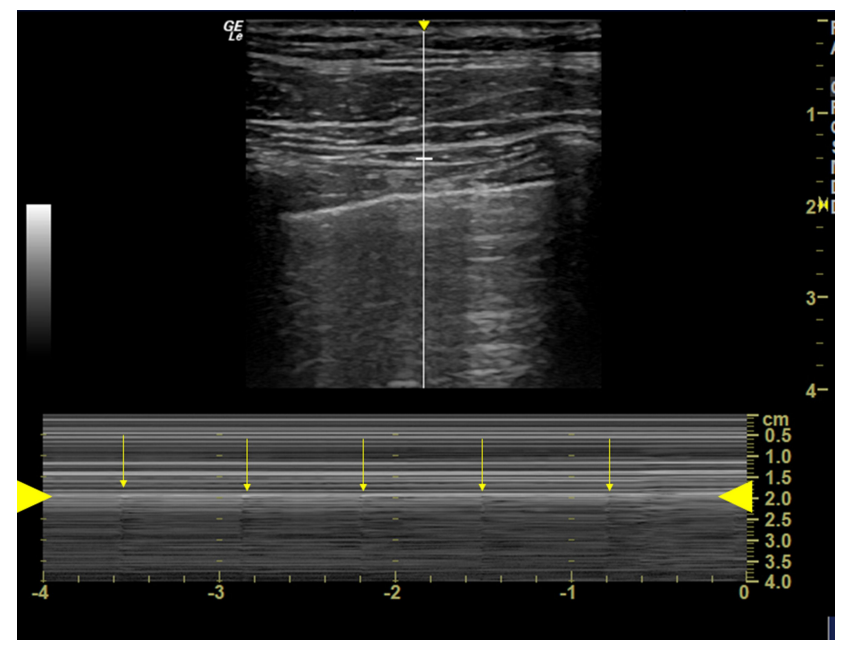

Figure 3 Bar code sign in an adult. M-mode point-of-care ultrasound. Numerous horizontal lines are seen both above and below the pleural line (between large yellow arrows). This is known as the bar code sign (stratosphere sign), which suggests the absence of lung sliding and the fact that the lung is not ventilated. Artifact created by the heart beat is detected at constant intervals (small yellow arrows).

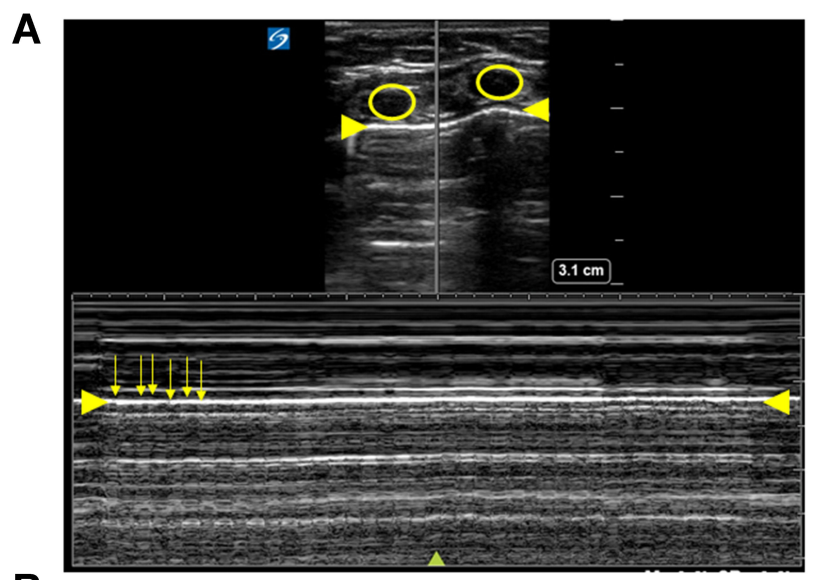

B

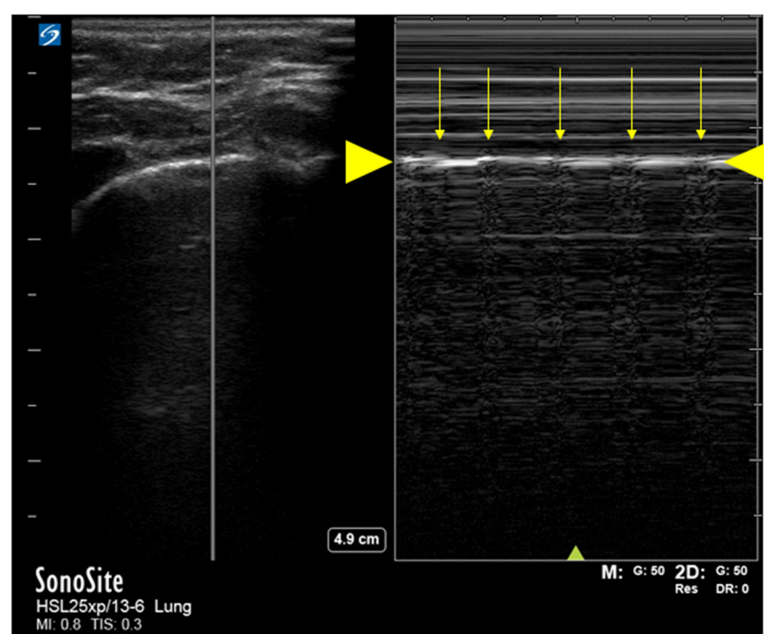

Figure 4 Bar code sign in an infant (A) and in a child (B). The ribs are the echoic areas within the yellow circles. The pleural line is noted by the larger yellow arrow. There are numerous artifacts in the bar code sign (small yellow arrows).

Subcutaneous emphysema may be present with a pneumothorax or after it is evacuated. The gas trapped in the subcutaneous tissue reflects ultrasound beams resulting in a high echoic line, known as an $\mathrm{E}$ line. ${ }^{17}$ The difference between $\mathrm{E}$ lines and pleural lines is the shape of the high echoic line and the places where the high echoic lines are generated. E lines are generated from subcutaneous tissue, which is more superficial and more scattered than pleural lines. When there is significant subcutaneous emphysema, the majority of the ultrasound beams are reflected at very shallow points with multiple artifacts, making it impossible to identify pleural lines. This process is referred to as "white out".

Many of the studies evaluating lung ultrasound were performed in patients who were scheduled for elective surgery with limited intrinsic lung disease. ${ }^{3-6}$ Pulmonary parenchymal with consolidation or atelectasis may impede lung ultrasound as lung sliding is observed only in ventilated areas. Therefore, even if two-lung ventilation is performed, 
areas with consolidation or atelectasis may impede identification of lung sliding. ${ }^{20,21}$ If pleural lines are not visible clearly below the ribs, atelectasis and consolidation may be present and other areas identified for scanning. ${ }^{18}$

\section{Summary}

Point-of-care ultrasound is an easy and powerful tool with potential applications during thoracic surgery in infants and children including confirmation of lung isolation during OLV. Lung sliding in the B-mode and the seashore sign in M-mode suggest that the lung is ventilated (Table 2). Pediatric anesthesiologists should be familiar with this technique because as it is not only non-invasive, but also more accurate when compared with auscultation.

\section{Ethics}

No IRB approval was required or obtained.

\section{Disclosure}

The authors declare no conflicts of interest.

\section{References}

1. Bataineh ZA, Zoeller C, Dingemann C, Osthaus A, Suempelmann R, Ure B. Our experience with single lung ventilation in thoracoscopic paediatric surgery. Eur J Pediatr Surg. 2012;22(1):17-20. doi:10.1055 s-0031-1285920

2. Ramsingh D, Frank E, Haughton R, et al. Auscultation versus point-of-care ultrasound to determine endotracheal versus bronchial intubation: a diagnostic accuracy study. Anesthesiology. 2016;124 (5):1012-1020.

3. Parab SY, Kumar P, Divatia JV, Sharma K. A prospective randomized controlled double-blind study comparing auscultation and lung ultrasonography in the assessment of double lumen tube position in elective thoracic surgeries involving one lung ventilation at a tertiary care cancer institute. Korean J Anesthesiol. 2019;72(1):24-31. doi:10. 4097/kja.d.17.00081

4. Hu WC, Xu L, Zhang Q, Wei L, Zhang W. Point-of-care ultrasound versus auscultation in determining the position of double-lumen tube. Medicine (Baltimore). 2018;97(13):e9311. doi:10.1097/MD.00000000 00009311

5. Alvarez-Diaz N, Amador-Garcia I, Fuentes-Hernandez M, DortaGuerra R. Comparison between transthoracic lung ultrasound and a clinical method in confirming the position of double-lumen tube in thoracic anaesthesia. A pilot study. Rev Esp Anestesiol Reanim. 2015; 62(6):305-312.
6. Parab SY, Divatia JV, Chogle A. A prospective comparative study to evaluate the utility of lung ultrasonography to improve the accuracy of traditional clinical methods to confirm position of left sided double lumen tube in elective thoracic surgeries. Indian J Anaesth. 2015;59 (8):476-481. doi:10.4103/0019-5049.162983

7. Yamaguchi Y, Moharir A, Nomura T, Tobias JD. Point-of-care lung ultrasound for bronchial blocker placement. A a Pract. 2019;13(7):287.

8. Nam JS, Park I, Seo H, Min HG. The use of lung ultrasonography to confirm lung isolation in an infant who underwent emergent video-assisted thoracoscopic surgery: a case report. Korean J Anesthesiol. 2015;68(4):411-414. doi:10.4097/kjae.2015.68.4.411

9. Yamaguchi Y, Moharir A, Burrier C, Tobias JD. Point-of-care lung ultrasound to evaluate lung isolation during one-lung ventilation in children: a case report. Saudi J Anaesth. 2019;13(3):243-245. doi:10.4103/sja.SJA_115_19

10. Adler AC. Extraluminal use of a pediatric bronchial blocker with confirmation by point-of-care ultrasound: a case report. A a Pract. 2019;13(1):13-16. doi:10.1213/XAA.0000000000000970

11. De Marchi L, Patel J, Razmjou K. Lung ultrasound in thoracic surgery: confirming placement of a pediatric right double-lumen tube. A a Pract. 2020;14(10):e01296. doi:10.1213/XAA.0000000000001296

12. Parab SY. Comment on the article "extraluminal use of a pediatric bronchial blocker with confirmation by point-of-care ultrasound". A a Pract. 2020;14(1):35. doi:10.1213/XAA.0000000000001121

13. Lichtenstein DA, Mezière GA. The BLUE-points: three standardized points used in the BLUE-protocol for ultrasound assessment of the lung in acute respiratory failure. Crit Ultrasound J. 2011;3 (2):109-110. doi:10.1007/s13089-011-0066-3

14. Ahn JH, Kwon E, Lee SY, Hahm TS, Jeong JS. Ultrasound-guided lung sliding sign to confirm optimal depth of tracheal tube insertion in young children. Br J Anaesth. 2019.

15. Lichtenstein DA, Lascols N, Prin S, Meziere G. The "lung pulse": an early ultrasound sign of complete atelectasis. Intensive Care Med. 2003;29(12):2187-2192. doi:10.1007/s00134-003-1930-9

16. Raimondi F, Rodriguez Fanjul J, Aversa S, et al. Lung ultrasound for diagnosing pneumothorax in the critically ill neonate. $J$ Pediatr. 2016;175:74-78.e71. doi:10.1016/j.jpeds.2016.04.018

17. Lichtenstein DA, Menu Y. A bedside ultrasound sign ruling out pneumothorax in the critically ill. Lung sliding. Chest. 1995;108 (5):1345-1348. doi:10.1378/chest.108.5.1345

18. Alrajhi K, Woo MY, Vaillancourt C. Test characteristics of ultrasonography for the detection of pneumothorax: a systematic review and meta-analysis. Chest. 2012;141(3):703-708. doi:10.1378/chest.11-0131

19. Francisco Neto MJ, Rahal Junior A, Vieira FAC, Silva PSDD, Funari MBDG. Advances in lung ultrasound. Einstein (São Paulo). 2016;14(3):443-448. doi:10.1590/S1679-45082016MD3557

20. Mallow C, Isakow W. Risk factors for loss of lung sliding in a medical intensive care population with acute respiratory failure. J Bronchology Interv Pulmonol. 2019;26(2):102-107. doi:10.1097/ LBR.0000000000000525

21. Liang H-Y, Liang X-W, Chen Z-Y, et al. Ultrasound in neonatal lung disease. Quant Imaging Med Surg. 2018;8(5):535-546. doi:10.21037/ qims.2018.06.01
Medical Devices: Evidence and Research is an international, peerreviewed, open access journal that focuses on the evidence, technology, research, and expert opinion supporting the use and application of medical devices in the diagnosis, monitoring, treatment and management of clinical conditions and physiological processes. The identification of novel devices and optimal use of existing devices which will lead to improved clinical outcomes and more effective patient management and safety is a key feature of the journal. The manuscript management system is completely online and includes a very quick and fair peer-review system. Visit http:// www.dovepress.com/testimonials.php to read real quotes from published authors. 